\title{
Improved Representation of Surface Spectral Emissivity in a Global Climate Model and Its Impact on Simulated Climate
}

\author{
Xianglei Huang, Xiuhong Chen, and Mark Flanner \\ Department of Climate and Space Sciences and Engineering, University of Michigan, Ann Arbor, Michigan \\ PING YANG \\ Department of Atmospheric Sciences, Texas A\&M University, College Station, Texas \\ DANIEL FELDMAN AND CHAINCY KUO \\ Division of Climate and Ecosystem Sciences, Lawrence Berkeley Laboratory, Berkeley, California
}

(Manuscript received 24 February 2017, in final form 26 January 2018)

\begin{abstract}
Surface longwave emissivity can be less than unity and vary significantly with frequency. However, most climate models still assume a blackbody surface in the longwave (LW) radiation scheme of their atmosphere models. This study incorporates realistic surface spectral emissivity into the atmospheric component of the Community Earth System Model (CESM), version 1.1.1, and evaluates its impact on simulated climate. By ensuring consistency of the broadband surface longwave flux across different components of the CESM, the top-of-the-atmosphere (TOA) energy balance in the modified model can be attained without retuning the model. Inclusion of surface spectral emissivity, however, leads to a decrease of net upward longwave flux at the surface and a comparable increase of latent heat flux. Global-mean surface temperature difference between the modified and standard CESM simulation is $0.20 \mathrm{~K}$ for the fully coupled run and $0.45 \mathrm{~K}$ for the slabocean run. Noticeable surface temperature differences between the modified and standard CESM simulations are seen over the Sahara Desert and polar regions. Accordingly, the climatological mean sea ice fraction in the modified CESM simulation can be less than that in the standard CESM simulation by as much as 0.1 in some regions. When spectral emissivities of sea ice and open ocean surfaces are considered, the broadband LW sea ice emissivity feedback is estimated to be $-0.003 \mathrm{~W} \mathrm{~m}^{-2} \mathrm{~K}^{-1}$, assuming flat ice emissivity as sea ice emissivity, and $0.002 \mathrm{~W} \mathrm{~m}^{-2} \mathrm{~K}^{-1}$, assuming coarse snow emissivity as sea ice emissivity, which are two orders of magnitude smaller than the surface albedo feedback.
\end{abstract}

\section{Introduction}

Surface emissivity $\varepsilon$ is defined as the ratio of actual surface emission to the blackbody radiation at the same temperature. It depends on surface properties such as composition, compactness of the medium, and surface roughness. As with other optical properties, the surface emissivity can vary with frequency and viewing angle. Since surface emissivity determines the amount of longwave radiation emitted by the surface, an incorrect representation of surface emissivity in the climate models may lead to an incorrect simulation of the surface energy budget and, therefore, potentially affect the overall simulated climate.

Corresponding author: Xianglei Huang, xianglei@umich.edu
Surface emissivity is indispensable in both the atmosphere and surface components of a global climate model (GCM) but many GCMs employ different representations of surface emissivity in their atmosphere and land models. For example, the standard Community Earth System Model (CESM) assumes a blackbody surface for all surface types in its atmosphere model, but graybody emissivity is assumed in the land model. Spectral variations of surface emissivities are not considered in either the atmosphere or land model components of the CESM. To ensure consistency of surface longwave flux across the atmosphere and land models, a radiative skin temperature is derived from the surface upward longwave flux generated from the land model and is then used in subsequent atmospheric radiative transfer calculation (further details are described in 
section 2a). We have also surveyed virtually all GCMs that participated in the latest IPCC assessment and found that, including the CESM, all but one GCM assume blackbody surface in the respective atmosphere components of the models.

In reality, the spectral variation of surface emissivity can be substantial. Figure 1 shows the spectral emissivities for water, ice, coarse snow, and desert surfaces, taken from the global surface spectral emissivity dataset developed by Huang et al. (2016). Different surface types exhibit different spectral dependence. For desert scenes, the spectral emissivity in the infrared window region can be lower than 0.8 . The spectral emissivity of water surface in part of the far-IR region $\left(0-200 \mathrm{~cm}^{-1}\right)$ is also lower than 0.9 .

The impact of such spectrally dependent surface emissivity on offline calculations of the radiative budget has been studied before. Chen et al. (2014) showed that, in comparison to the blackbody surface assumption, taking the far-IR surface emissivity of snow into account can affect the monthly mean polar radiation budget at the top of the atmosphere (TOA). Huang et al. (2016) developed a global surface spectral emissivity dataset based on a hybrid approach using both first-principle calculations and satellite retrievals of surface spectral emissivity. Huang et al. (2016) also showed the nonnegligible impact of such surface emissivity on the offline calculation of the TOA longwave (LW) radiation budget. Compared to the blackbody surface assumption, using realistic surface emissivity reduces the globally averaged outgoing longwave radiation (OLR) by about $0.7 \mathrm{~W} \mathrm{~m}^{-2}$ and the regional difference of OLR can be as large as $-10 \mathrm{~W} \mathrm{~m}^{-2}$, as shown by Huang et al. (2016). In addition, Feldman et al. (2014) suggested that the far-IR surface emissivity could also impact the simulated climate changes by the CESM in response to an increase of atmospheric $\mathrm{CO}_{2}$. Therefore, a meaningful question worth further investigation is the following: If spectrally dependent surface emissivity is correctly incorporated into the climate model simulations, to what extent can this incorporation affect simulated climatology and climate changes? Our study attempts to use the CESM to answer this question.

The remaining sections are arranged as follows. Section 2 introduces the CESM, the primary modeling tool used in our study, and describes modifications to the CESM that facilitate spectrally varying surface emissivity. Section 3 presents the impact of incorporating surface spectral emissivity in the CESM on its simulated mean climate state. The possible sea ice emissivity feedback in response to the doubling of $\mathrm{CO}_{2}$ is discussed in section 4. Conclusions and further discussions are then given in section 5 .

\section{Model, modification, and simulation setup}

\section{a. The CESM model}

This study uses the CESM, version 1.1.1, developed by the National Center of Atmospheric Research (NCAR). Details about the CESM can be found in Gent et al. (2011). The CESM contains six models, namely, models of atmosphere, ocean, land, sea ice, land ice, and river runoff, as well as a coupler that exchanges information among the six models. The atmosphere model has a horizontal resolution of $1.9^{\circ}$ latitude by $2.5^{\circ}$ longitude and 26 layers in the vertical. It employs the longwave version of the Rapid Radiative Transfer Model for GCMs (RRTMG_LW; Mlawer et al. 1997; Clough et al. 2005) for its longwave radiative scheme. The RRTMG_LW was adapted from the longwave version of the RRTM for improved efficiency in GCMs. It has 16 wavelength bands from 10 to $3250 \mathrm{~cm}^{-1}$ and, in the standard CESM, surface emissivity is always assumed to be unity for all bands in the RRTMG_LW. Cheng et al. (2016) quantified the error of RRTMG_LW when spectrally dependent surface emissivity is used in the calculation. They concluded that, compared to benchmark line-by-line radiative transfer calculations, the errors in radiative cooling rate computed by the RRTMG_LW are confined to the lowest part of the troposphere and range from -0.23 to $0.19 \mathrm{~K} \mathrm{day}^{-1}$.

Land surface and ocean models in the CESM assume graybody emissivities (i.e., surface emissivities are constant over all the frequencies but less than unity) (Neale et al. 2012). The land model assumes an emissivity of 0.97 for snow and nonurban ground, 0.96 for urban ground, and a predefined value for vegetation with different leaf area index (LAI). Snow and ice emissivity is 0.98 in the sea ice model. Ocean surface is assumed to be a blackbody in the CESM. The broadband upward LW flux $F_{\mathrm{LW}}^{\uparrow}$ is first computed by the land, ice, or ocean models. A radiative skin temperature $T_{\text {skin }}$ is then defined assuming the surface is a blackbody, that is,

$$
T_{\text {skin }}=\left(\frac{F_{\mathrm{LW}}^{\uparrow}}{\sigma}\right)^{1 / 4},
$$

where $\sigma$ is the Stefan-Boltzmann constant. The value for $T_{\text {skin }}$ is then used in the RRTMG_LW calculation within the atmosphere model. Therefore, even if the surface emissivity is treated differently in the atmosphere and surface components of the CESM, $F_{\mathrm{LW}}^{\uparrow}$ is ensured to be identical across these models, which is necessary for energy conservation. Similarly, any modifications to the CESM for inclusion of surface spectral emissivity also need to maintain consistency of $F_{\mathrm{LW}}^{\uparrow}$ across the atmosphere and surface model components. 


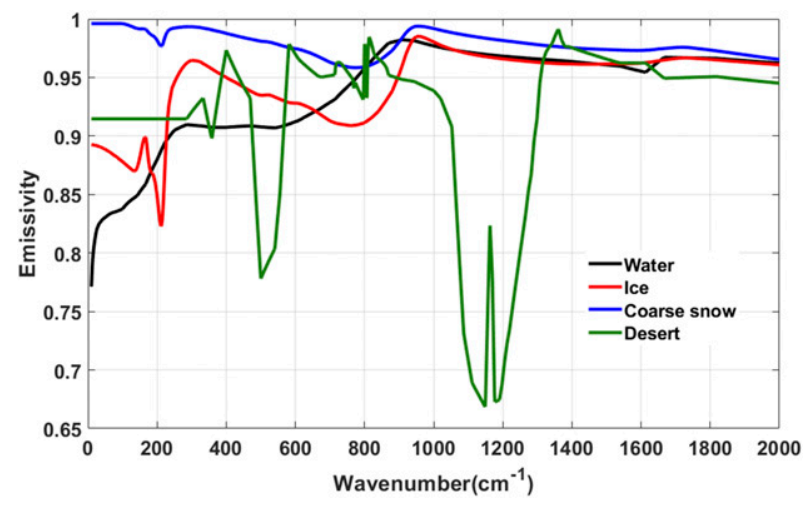

FIG. 1. The spectral emissivities of water, ice, coarse snow, and desert. The emissivities are derived using appropriate radiative transfer techniques and the mid-IR portions of the emissivities have been validated against available measurements. Further details can be found in Huang et al. (2016).

This is a prerequisite for the modified CESM to be used for understanding the impact of such inclusion of surface spectral emissivity on simulated climatology and climate changes.

\section{b. Incorporating surface spectral emissivity into the atmosphere model of the CESM}

We denote the emissivity for the $i$ th band in the RRTMG_LW as $\varepsilon_{i}$. Then, the upward LW broadband flux at the surface is the sum of surface emission and reflection of downward flux, given by

$$
F_{\mathrm{LW}}^{\uparrow}=\sum_{i} F_{i}^{\uparrow}=\sum_{i} \varepsilon_{i} \pi \int_{\Delta v_{i}} B_{v}\left(T_{\mathrm{skin}}\right) d v+\sum_{i}\left(1-\varepsilon_{i}\right) F_{\mathrm{sfc}, i}^{\downarrow},
$$

where $B_{v}\left(T_{\text {skin }}\right)$ is Planck's function, $\Delta v_{i}$ is the bandwidth of the $i$ th band in the RRTMG_LW, $F_{i}^{\uparrow}$ is the surface upward flux of the $i$ th band, and $F_{\text {sfc, } i}^{\downarrow}$ is the surface downward flux of the $i$ th band at the surface as computed by the RRTMG_LW. With $F_{\mathrm{LW}}^{\uparrow}$ from the land or ocean or ice models, $F_{\mathrm{scc}, i}^{\downarrow}$ from the atmosphere model, and approximating the integral in Eq. (2) in terms of a Gaussian quadrature, $T_{\text {skin }}$ can be iteratively solved. In this form, the broadband upward LW flux at surface entering RRTMG_LW calculations is ensured to be the same as the counterpart calculated in the other model components of the CESM, up to numerical errors of the numeric solver for Eq. (2). Hereafter, we use the term modified CESM to refer to the model with such modifications as described above.

As a verification, we run the modified CESM with $\varepsilon_{i}=1$ for all RRTMG_LW bands and all surface types. The simulation should be the same as the counterpart using the standard CESM because both assume blackbody surfaces in the RRTMG_LW. The difference between the two runs should be caused only by the numerical errors in solving Eq. (2) for $T_{\text {skin }}$, because Eq. (2) is the exact analytical solution for $T_{\text {skin }}$. The modified CESM with $\varepsilon_{i}=1$ and the standard CESM are initialized with the same initial conditions and run for 40 years. Figures $2 \mathrm{a}$ and $2 \mathrm{~b}$ show the snapshot differences in $T_{\text {skin }}$ and $F_{\mathrm{LW}}^{\uparrow}$ at the surface after $3 \mathrm{~h}$ of simulation (i.e., 6 time steps of integration), respectively. As expected, the differences between the two simulations are small. Differences in the time series of globally and annually averaged $T_{\text {skin }}$ and $F_{\mathrm{LW}}^{\uparrow}$ at the surface are shown in Figs. $2 \mathrm{c}$ and $2 \mathrm{~d}$, respectively. Over the course of 40 years of simulations, the difference between annualand global-mean $T_{\text {skin }}$ is within $\pm 0.2 \mathrm{~K}$, and the difference between annual- and global-mean $F_{\mathrm{LW}}^{\uparrow}$ at the surface is within $\pm 1 \mathrm{~W} \mathrm{~m}^{-2}$. If we use averages from simulation years 6-35 to form a 30-yr climatological mean difference, the difference in global-mean $T_{\text {skin }}$ is only $-0.05 \mathrm{~K}$, and the difference in global-mean $F_{\mathrm{LW}}^{\uparrow}$ at the surface is only $-0.17 \mathrm{~W} \mathrm{~m}^{-2}$, as expected from the scaling with $1 / \sqrt{N}$ where $N$ is the number of years used to form the average. These results verify that our modification to the CESM can maintain consistency of the broadband $F_{\mathrm{LW}}^{\uparrow}$ across different model components of the CESM.

We note that this study only incorporates the surface spectral emissivity into the atmosphere model of the CESM. The treatment of surface emissivity in the other parts of the CESM remains unchanged. Eventually, in order to faithfully represent the surface radiative process, radiative schemes in other components of the CESM should be modified to include the surface spectral emissivities as well.

\section{c. Global spectral surface emissivity dataset}

This study makes use of a monthly mean global surface spectral emissivity dataset developed by Huang et al. (2016). The dataset contains spectral emissivities over the entire LW spectrum for 11 surface types: water, fine snow, medium snow, coarse snow, ice, grass, dry grass, conifer, deciduous forest, desert, and a combination of $55 \%$ vegetation and $45 \%$ desert. The emissivities of water and ice surfaces are computed using the Fresnel equations. The emissivities of snow surfaces are computed following the methods described by Chen et al. (2014). The emissivities of the four types of vegetation are taken directly from measurements compiled in the Advanced Spaceborne Thermal Emission Reflection Radiometer (ASTER) Spectral Library 2.0 (Baldridge et al. 2009). The emissivities of desert are computed assuming that the desert surface is composed of coarse sand grains and fine silts. As a result, 16 subtypes of 
(a)

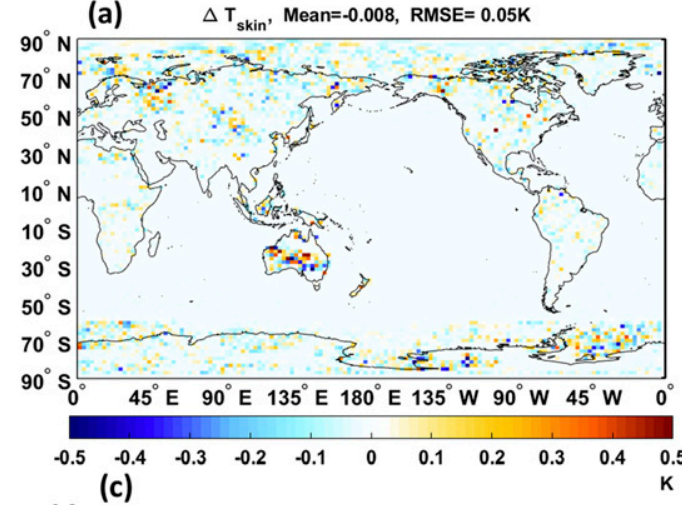

(b) $\triangle$ LW flux $@$ Surface, Mean $=-0.02$, RMSE $=0.06 \mathrm{Wm}^{-2}$

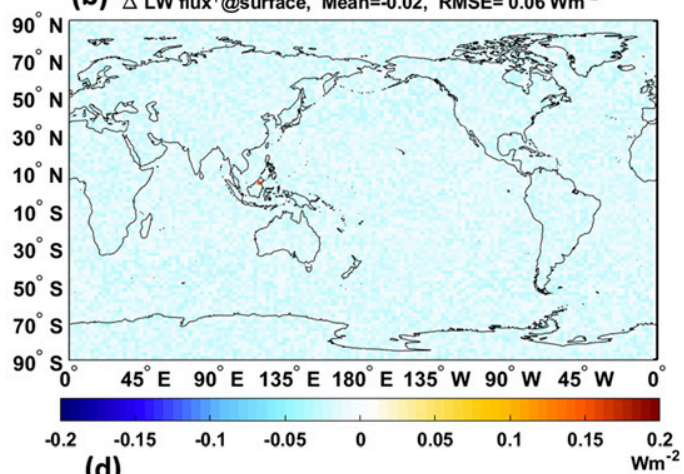

(d)
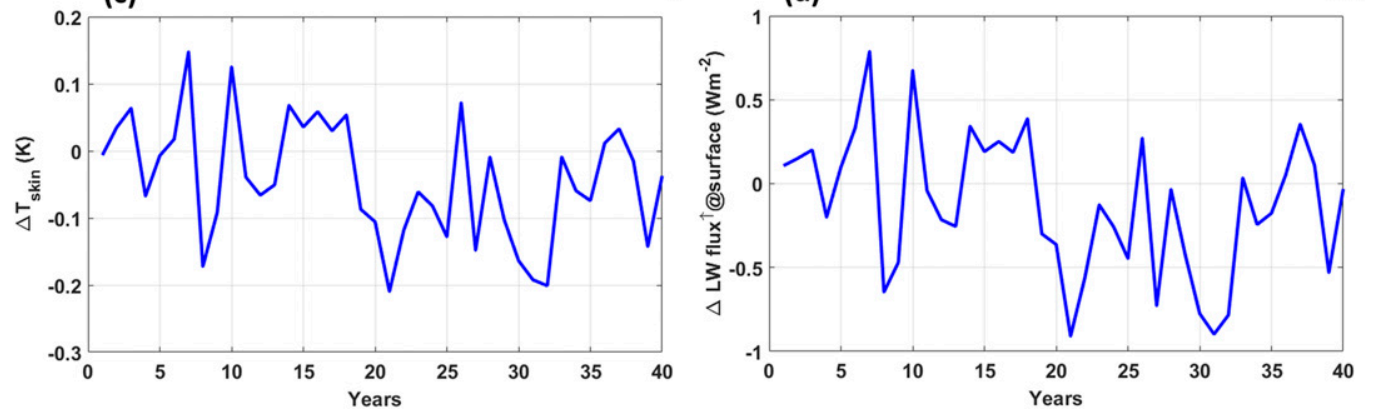

FIG. 2. (a) The snapshot differences of surface skin temperature at the sixth time step (i.e., $3 \mathrm{~h}$ of simulation) between two simulations. One simulation uses the modified CESM but assuming $\varepsilon_{i}=1$ for all surface type and for all RRTMG_LW band (i.e., blackbody surface), and the other simulation uses the standard CESM. Two simulations are initialized with the same initial conditions. (b) As in (a), but for the surface upward LW flux. (c) Globally averaged annual-mean difference of $T_{\text {skin }}$ between two simulations from year 1 to 40. (d) As in (c), but for the surface upward LW flux.

desert surfaces were defined in order to fit the observed range of desert surface spectral emissivities.

To obtain the gridded surface spectral emissivity for the entire globe, the aforementioned spectral emissivities of 11 different surface types (including subtypes) are regressed against monthly mean surface spectral emissivity at 8 discrete mid-IR frequencies as retrieved from MODIS (Seemann et al. 2008) at high spatial resolution $\left(0.05^{\circ} \times 0.05^{\circ}\right)$. Huang et al. (2016) validated the gridded surface spectral emissivity at $0.5^{\circ} \times 0.5^{\circ}$ spatial resolution with respect to the surface spectral emissivity retrieved from the Infrared Atmospheric Sounding Interferometer (IASI; Zhou et al. 2011). In this study, over land surfaces, we spatially average the surface spectral emissivity dataset of Huang et al. (2016) onto the CESM grid $\left(1.9^{\circ} \times 2.5^{\circ}\right)$ and spectrally average them onto the RRTMG_LW spectral grid. Over desert and its adjacent areas, if the LAI for a particular month is smaller than 0.001, the desert spectral emissivity is then used. If LAI is larger than 2 for a month, the grass spectral emissivity is used. For a grid cell with LAI between 0.001 and 2, we then use the surface spectral emissivity for the same calendar month in the dataset developed by Huang et al. (2016). For ocean grid cells, the water surface spectral emissivity is averaged onto the RRTMG_LW bands and then used in the model. For grid cells with sea ice coverage, the surface spectral emissivity is taken as the average of ice and water spectral emissivities, weighted by the sea ice and open ocean fractions. As an example, Fig. 3 shows the surface emissivity map for July of simulation year 1 , for two RRTMG_LW bands, $350-500 \mathrm{~cm}^{-1}$ (a far-IR band) and $1080-1180 \mathrm{~cm}^{-1}$ (a mid-IR window band). The surface emissivity of desert can be as low as 0.8 in the mid-IR window band, and the surface emissivity of open water can be also as low as 0.9 in the far-IR band (Fig. 3).

\section{d. Simulation designs}

To assess the impact of incorporating surface spectral emissivity in the CESM on the simulated climate, we use both the standard CESM and modified CESM to perform simulations in parallel, initialized with the same initial conditions. Figure 4 shows the time series of simulated globally and annually averaged TOA energy imbalance for both the slab-ocean run (Fig. 4, top) and fully coupled run (Fig. 4, bottom). After a few years of spinup, the modified CESM reaches a similar TOA energy balance as observed in the standard CESM. In the 
(a) $350-500 \mathrm{~cm}^{-1}$

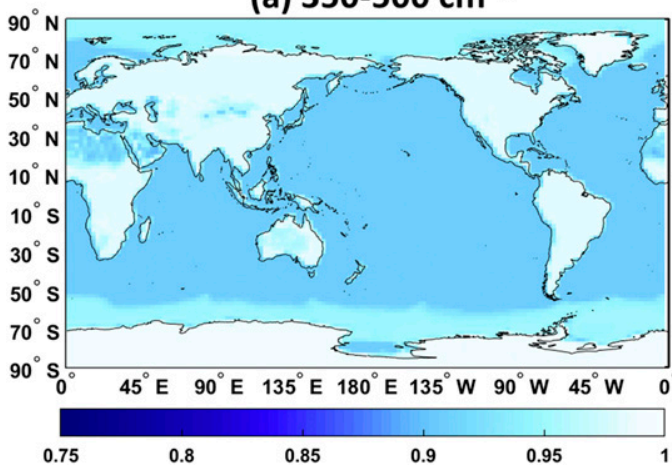

(b) $1080-1180 \mathrm{~cm}^{-1}$

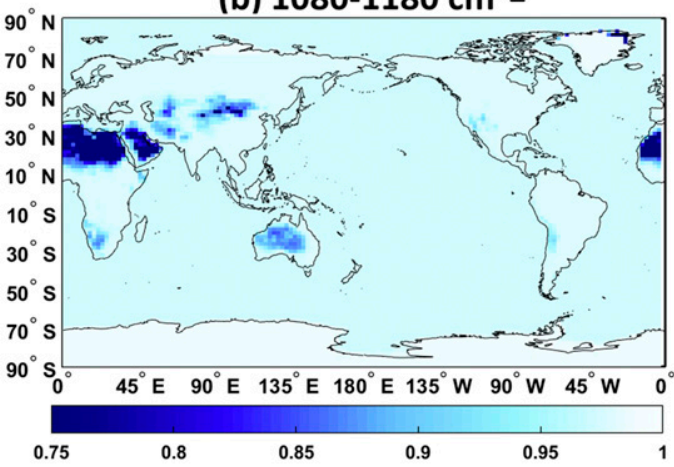

FIG. 3. (a) The emissivity used for the RRTMG_LW band $350-550 \mathrm{~cm}^{-1}$ in July of year 1 by the modified CESM. (b) As in (a), but for the RRTMG_LW band $1080-1180 \mathrm{~cm}^{-1}$.

case of the slab-ocean run, the TOA imbalance averaged over years $6-35$ is $0.03 \mathrm{~W} \mathrm{~m}^{-2}$ for the standard CESM and $-0.03 \mathrm{~W} \mathrm{~m}^{-2}$ for the modified CESM. In the case of the fully coupled run, the TOA imbalance for years 6-35 is $-0.07 \mathrm{~W} \mathrm{~m}^{-2}$ for the standard CESM and $-0.29 \mathrm{~W} \mathrm{~m}^{-2}$ for the modified CESM. For the modified CESM, TOA imbalance statistics change little if another 30-yr time period is examined, as long as the starting year is after the fifth year of the simulation. Therefore, our modification to the CESM, which ensures broadband LW flux at the surface to be the same across different model components of the CESM, does not significantly impact the simulated TOA imbalance and there is no need to retune the CESM to achieve TOA energy balance.

Unless noted otherwise, the following analyses of simulated climatology differences between the standard CESM and modified CESM are based on 30-yr averages of the simulations (years 6-35). Analyses using other periods of 30 years give essentially the same results.
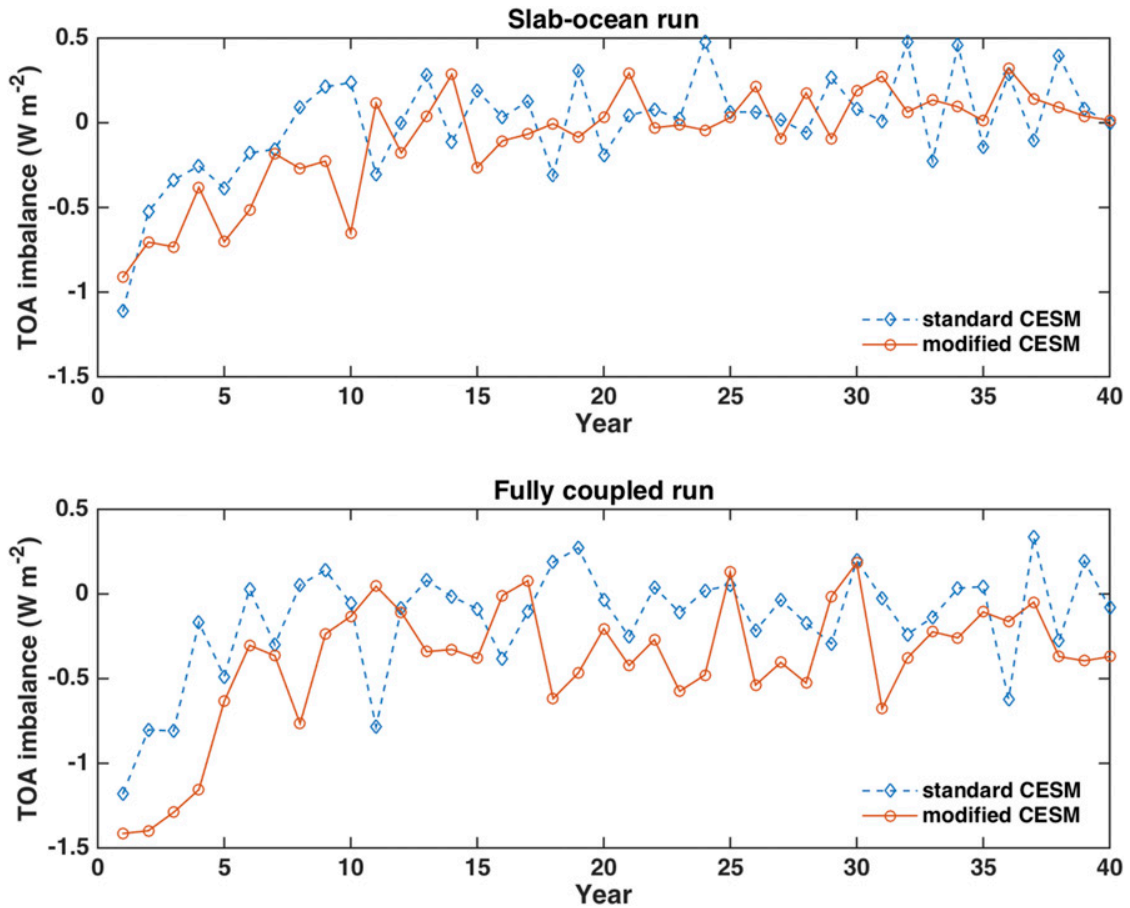

FIG. 4. (top) Time series of annual-mean TOA energy imbalance (upward positive) as simulated by the standard CESM (blue dashed line) and the modified CESM (red solid line). Both are slab-ocean runs with the same initial conditions. (bottom) As in (top), but for the fully coupled runs. 
Both slab-ocean and fully coupled simulations are analyzed in this study. Oceans only have thermodynamic responses to the imposed changes in the slab-ocean runs while they can have both dynamic and thermodynamic responses to the imposed changes in the fully coupled runs. Therefore, the differences between the slab-ocean and fully coupled runs can help us understand to what extent ocean dynamics respond to the surface emissivity changes that we imposed.

In addition to investigating the impact on simulated climatology, this study also assesses the possible sea ice emissivity feedback in response to a doubling of $\mathrm{CO}_{2}$. Details of simulation design for this assessment will be given in section 4, together with the assessment results.

Observations show that sea ice surfaces are rough and can have a surface granular layer, which resembles coarse-grained snow (Petrich and Eicken 2009). As shown in Fig. 1, the spectral dependencies of flat ice surface and coarse-grained snow emissivities are different. The emissivity of coarse snow is larger than that of water for all frequencies except a small portion of 800 $900 \mathrm{~cm}^{-1}$, where it is only slightly smaller than water surface emissivity. The emissivity of flat ice, however, is larger than that of water for the majority of the far-IR but noticeably smaller than that of water for $650-950 \mathrm{~cm}^{-1}$. To understand how such different assumptions of sea ice emissivity can affect the simulation, we also carry out simulations assuming that the sea ice emissivity is the same as the coarse snow emissivity, while the rest of the simulation designs are identical to the aforementioned ones. The results will also be discussed in sections 3 and 4 .

\section{e. A heuristic argument about surface emissivity and surface upward longwave flux}

To facilitate the interpretation of model simulation results between the modified and standard CESM, it is worthwhile to take a close look at Eq. (2), the expression for upward LW flux at surface. For each spectral band, the upward longwave flux at surface $F_{i}^{\uparrow}$ consists of two components: the term of direct emission from surface, $\varepsilon_{i} \pi \int_{\Delta v_{i}} B_{v}\left(T_{\text {skin }}\right) d v$, and the term of reflection of downward flux, $\left(1-\varepsilon_{i}\right) F_{\text {sfc }, i}^{\downarrow}$. The responses of these two components to the change of surface emissivity are opposite. If $\varepsilon_{i}$ increases and everything else remains unchanged, the surface emission will increase but the reflection of downward flux will decrease. There are two scenarios particularly relevant to the discussion:

1) If $F_{\text {sfc, }, i}^{\downarrow} \cong \pi \int_{\Delta v_{i}} B_{v}\left(T_{\text {skin }}\right) d v$ (i.e., the downward flux in the $i$ th band $F_{\text {sfc } i}^{\downarrow}$ is nearly the same as the blackbody emission over the spectral band for the surface skin temperature), then we have $\varepsilon_{i} \pi \int_{\Delta v_{i}} B_{v}\left(T_{\text {skin }}\right) d v-\varepsilon_{i} F_{\text {sfc, } i}^{\downarrow} \cong 0$ regardless of the value of $\varepsilon_{i}$. Then we always have $F_{i}^{\uparrow} \cong F_{\text {sfc, } i}^{\downarrow} \cong$ $\pi \int_{\Delta v_{i}} B_{v}\left(T_{\text {skin }}\right) d v$ (i.e., $F_{i}^{\uparrow}$ having little dependence on the actual value of $\varepsilon_{i}$ ). This scenario applies to the spectral bands where the atmospheric opacity is so large that the effective emission level for downward flux is close to the surface (e.g., the majority of the $\mathrm{CO}_{2} \mathrm{v}_{2}$ band). For the far-IR rotational band and the $\mathrm{v}_{2}$ band of $\mathrm{H}_{2} \mathrm{O}$, this scenario also applies to the vast majority of the globe, as long as the atmosphere is not extremely dry (Chen et al. 2014).

2) If $F_{\mathrm{sfc}, i}^{\downarrow}$ is much smaller than $\pi \int_{\Delta v_{i}} B_{v}\left(T_{\text {skin }}\right) d v$, then the change to the surface emission term caused by any change of $\varepsilon_{i}$ will be larger than the corresponding change of the reflection term. Therefore, the larger the deviation of $\varepsilon_{i}$ from unity, the larger the change of $F_{i}^{\uparrow}$ compared to the case of blackbody surface. The total optical depth in the IR window band is much less than 1 and, therefore, $F_{\text {sfc, } i}^{\downarrow}$ is small and the effective emission level is usually in the upper troposphere or even higher. The surface emissivity over deserts is around 0.8 or even lower over the IR window band (Fig. 3b). Therefore, this scenario applies to the IR window band, especially for desert regions. The scenario also applies to the far-IR spectral bands over high-elevation mountain and polar regions, where the atmosphere column water vapor is small (Chen et al. 2014).

\section{Differences in simulated climatology between the modified and standard CESM}

\section{a. Differences in the globally averaged energy budget}

The global-mean energy budget from the standard CESM simulation is summarized in Table 1, as well as the differences between the modified and standard CESM simulations. The modified - standard CESM differences in the surface energy budget can be summarized as follows:

1) The net longwave flux [upward ( $\mathrm{LW}$ flux $\uparrow)$ - downward (LW flux $\downarrow$ )] at surface changes by $-0.90 \mathrm{~W} \mathrm{~m}^{-2}$ for the slab-ocean run and $-0.79 \mathrm{~W} \mathrm{~m}^{-2}$ for the fully coupled run. This decrease in the net upward LW flux is largely compensated for by an increase in latent heat flux, which is $1.01 \mathrm{~W} \mathrm{~m}^{-2}$ for the slab-ocean run and $0.59 \mathrm{~W} \mathrm{~m}^{-2}$ for the fully coupled run. The standard errors calculated using annual-mean differences are much smaller than these changes. These changes are also much larger than $-0.17 \mathrm{~W} \mathrm{~m}^{-2}$, the 30 -yr average of the surface LW flux change when the blackbody surface is used in the modified CESM simulation (Fig. 2d). Therefore, the changes in LW flux and latent heat shown here can be confidently 
TABLE 1. Simulated global-mean energy budget by the standard CESM as well as the differences between the modified CESM and the standard CESM. Results are based on 30 years of averages. Energy imbalance, latent heat, and sensible heat flux are all defined positive upward. Difference is expressed in terms of 30-yr mean difference plus or minus the standard error computed using annual-mean differences. Surface temperature here refers to surface air temperature over land and SST over ocean, the same definition as used by the observational surface temperature datasets.

\begin{tabular}{|c|c|c|c|c|}
\hline & \multicolumn{2}{|r|}{ Slab-ocean run } & \multicolumn{2}{|r|}{ Fully coupled run } \\
\hline & $\begin{array}{l}\text { Standard } \\
\text { CESM }\end{array}$ & $\begin{array}{c}\text { Difference } \\
\text { (modified }- \text { standard CESM) }\end{array}$ & $\begin{array}{l}\text { Standard } \\
\text { CESM }\end{array}$ & $\begin{array}{c}\text { Difference } \\
\text { (modified }- \text { standard CESM) }\end{array}$ \\
\hline \multicolumn{5}{|l|}{ Surface energy budget } \\
\hline $\mathrm{LW}$ flux $\uparrow\left(\mathrm{W} \mathrm{m}^{-2}\right)$ & 401.16 & $2.26 \pm 0.07$ & 400.83 & $1.00 \pm 0.16$ \\
\hline $\mathrm{LW}$ flux $\downarrow\left(\mathrm{W} \mathrm{m}^{-2}\right)$ & 344.56 & $3.16 \pm 0.10$ & 343.95 & $1.79 \pm 0.19$ \\
\hline SW flux $\uparrow\left(\mathrm{W} \mathrm{m}^{-2}\right)$ & 22.85 & $-0.38 \pm 0.02$ & 22.72 & $-0.17 \pm 0.04$ \\
\hline $\mathrm{SW}$ flux $\downarrow\left(\mathrm{W} \mathrm{m}^{-2}\right)$ & 181.39 & $-0.44 \pm 0.06$ & 181.41 & $-0.27 \pm 0.08$ \\
\hline Latent heat flux $\left(\mathrm{W} \mathrm{m}^{-2}\right)$ & 83.41 & $1.01 \pm 0.05$ & 83.09 & $0.59 \pm 0.07$ \\
\hline Sensible heat flux $\left(\mathrm{W} \mathrm{m}^{-2}\right)$ & 17.97 & $-0.20 \pm 0.02$ & 18.03 & $-0.10 \pm 0.03$ \\
\hline Energy imbalance $\left(\mathrm{W} \mathrm{m}^{-2}\right)$ & -0.56 & $-0.03 \pm 0.04$ & -0.69 & $-0.20 \pm 0.06$ \\
\hline \multicolumn{5}{|l|}{ TOA energy budget } \\
\hline $\mathrm{LW}$ flux $\uparrow\left(\mathrm{W} \mathrm{m}^{-2}\right)$ & 235.17 & $0.19 \pm 0.04$ & 235.08 & $-0.15 \pm 0.07$ \\
\hline $\mathrm{SW}$ flux $\uparrow\left(\mathrm{W} \mathrm{m}^{-2}\right)$ & 106.67 & $-0.25 \pm 0.05$ & 106.66 & $-0.06 \pm 0.06$ \\
\hline Energy imbalance $\left(\mathrm{W} \mathrm{m}^{-2}\right)$ & 0.03 & $-0.06 \pm 0.05$ & -0.07 & $-0.21 \pm 0.06$ \\
\hline \multicolumn{5}{|l|}{ Others } \\
\hline Net column radiative cooling $\left(\mathrm{W} \mathrm{m}^{-2}\right)$ & 103.36 & $0.78 \pm 0.04$ & 103.13 & $0.47 \pm 0.07$ \\
\hline$T_{\text {skin }}(\mathrm{K})$ & 288.68 & $0.78 \pm 0.01$ & 288.63 & $0.54 \pm 0.03$ \\
\hline$T_{s}(\mathrm{~K})$ & 288.53 & $0.45 \pm 0.01$ & 288.48 & $0.20 \pm 0.03$ \\
\hline Precipitation (mm day ${ }^{-1}$ ) & 2.88 & $0.03 \pm 0.002$ & 2.870 & $0.02 \pm 0.003$ \\
\hline
\end{tabular}

attributed to the altered surface emissivity treatment, rather than any statistical fluctuations of the model simulation.

2) The changes in shortwave flux at the surface [both upward (SW flux $\uparrow$ ) and downward (SW flux $\downarrow$ )] and sensible heat flux are both much smaller than the changes in net upward longwave flux and latent heat flux.

3) The net surface energy imbalance is changed by -0.03 and $-0.2 \mathrm{~W} \mathrm{~m}^{-2}$ for the slab-ocean run and the fully coupled run, respectively. These changes are much smaller than the 30-yr average of surface energy imbalance in the standard CESM, which is $-0.56 \mathrm{~W} \mathrm{~m}^{-2}$ for the slab-ocean run and $-0.69 \mathrm{~W} \mathrm{~m}^{-2}$ for the fully coupled run. Therefore, incorporating surface spectral emissivity into the CESM does not significantly affect the simulated surface energy imbalance.

The changes in outgoing longwave and shortwave fluxes at the TOA are small in magnitude $\left(\sim 0.2 \mathrm{~W} \mathrm{~m}^{-2}\right.$ or even less). Therefore, inclusion of surface spectral emissivity in the CESM has little impact on the energy imbalance at the TOA, as already shown in Fig. 4 and discussed in section $2 \mathrm{~d}$. The net column radiative cooling of the entire atmosphere, as a result, is $0.78 \mathrm{~W} \mathrm{~m}^{-2}$ $\left(0.47 \mathrm{~W} \mathrm{~m}^{-2}\right)$ larger in the modified CESM slab-ocean (fully coupled) run than in the standard CESM counterpart run. Correspondingly, global precipitation increases slightly in accord with the change in atmospheric column radiative cooling and the change in sensible heat flux (loss of sensible heat from the surface). For example, for the fully coupled run, the total change of diabatic cooling of the atmosphere due to radiation and sensible heat transfer is $0.57 \mathrm{~W} \mathrm{~m}^{-2}$. With a latent heat of $2.5 \times 10^{6} \mathrm{~J} \mathrm{~kg}^{-1}$, a change of precipitation by $0.0197 \mathrm{~mm} \mathrm{day}^{-1}$ is needed to balance this change in diabatic cooling. This estimate is in excellent agreement with the $0.02 \mathrm{~mm} \mathrm{day}^{-1}$ change seen in the actual simulations (Table 1).

Figure 5 shows the difference of globally averaged temperature profile between the modified and standard CESM simulations, as well as the spatial map of the difference at $850 \mathrm{hPa}$. The temperature difference is constant throughout the free troposphere and the spatial distribution is also homogenous. Cheng et al. (2016) show that the direct impact of including surface spectral emissivity on atmospheric radiative heating rate is limited to the boundary layer. These facts indicate that including spectral surface emissivity has little impact on the atmospheric lapse rate in the free troposphere. Near the surface, the air temperature change has a peak in the boundary layer for both slab-ocean and fully coupled runs (Fig. 5a). The positive air temperature change for the global-mean profile (Fig. 5a) is consistent with the increase of downward LW flux at surface shown in Table 1. We think that Table 1 can be explained as follows: since the surface spectral emissivity is less than unity in the 
(a)

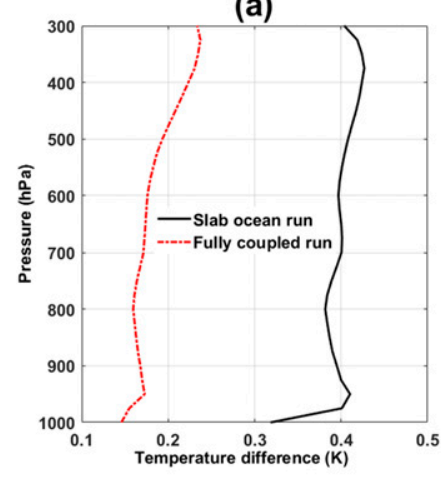

(b)

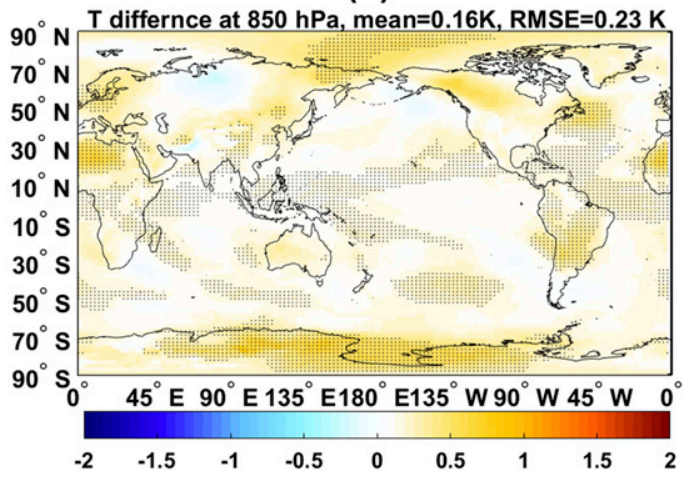

FIG. 5. (a) The difference in 30-yr climatology of global-mean temperature profiles from 1000 to $300 \mathrm{hPa}$ between the modified and the standard CESM simulations. The black line is for the slab-ocean run and the red line for the fully coupled run. (b) The map of temperature difference at $850 \mathrm{hPa}(\mathrm{K})$. Results from fully coupled runs are shown here and the stippling shows areas with statistically significant differences ( $5 \%$ significance level). The difference at other pressure levels in the free troposphere has similar spatial distribution as shown here.

modified CESM, the change in the surface emission term, compared with the default model, will dominate over the change in the reflection of downward flux term (section 2e), and thus the total upward LW flux would be reduced if everything else remained unchanged. However, the increased surface temperature compensates for this reduction. Meanwhile, the atmospheric lapse rate is affected little by the change of surface emissivity, as argued above and shown in Fig. 5. Therefore, an increase of surface temperature leads to an increase of air temperature above it. The higher air temperature everywhere above the surface then leads to a larger downward LW flux at the surface. The overall result is that, compared to the standard CESM simulation, the modified CESM has an increase in net downward LW flux, and correspondingly, an increase in upward latent heat flux for maintaining the surface energy balance.

The difference in global-mean surface skin temperature between the modified and standard CESM is $0.78 \mathrm{~K}$ for the slab-ocean run and $0.54 \mathrm{~K}$ for the fully coupled run. Given that the surface skin temperature is a radiative quantity coupled with the definition of surface emissivity, it is meaningful to examine the surface temperature that is normally used in observation-model comparisons. Following the convention used in compiling observational surface temperature data, we use surface air temperature (SAT; temperature at $2 \mathrm{~m}$ above the surface) over land and sea surface temperature over ocean for such comparison. For brevity, hereafter we will refer to this combined temperature field as surface temperature $T_{s}$. The difference in global-mean surface temperature is $0.45 \mathrm{~K}$ for the slab-ocean run and $0.20 \mathrm{~K}$ for the fully coupled run (Table 1). As discussed in section $3 b$, although the global-mean surface air temperature difference is small, some regional differences are much larger.

\section{b. Spatial distributions and seasonality of the differences}

The differences in surface temperatures $\Delta T_{s}$ between the modified and standard CESM runs are shown in Figs. 6a,c,e. The difference between modified and standard CESM runs is ubiquitously nonnegative. The majority of such positive differences are also statistically significant, passing the Student's $t$ test at a 5\% significance level (annual-mean differences are used in the test). For the slab-ocean run, positive difference of approximately $1-2 \mathrm{~K}$ can be seen over the Sahara Desert region as well as high latitudes of both hemispheres. The pattern of $\Delta T_{s}$ from the fully coupled run is similar to that from the slab-ocean run, with smaller positive difference over the Southern Hemisphere polar regions. For both runs, $\Delta T_{s}$ is overwhelmingly nonnegative. Consistent with the heuristic arguments in section 2e, noticeable differences are seen over the regions where the changes in surface emissivities alone impact the surface upward longwave flux the most (i.e., the desert where window region surface emissivity is much less than unity and downward LW flux in the window region is small, and the high latitudes where cold and dry atmosphere also leads to small downward LW flux in the $\mathrm{H}_{2} \mathrm{O}$ bands; Chen et al. 2014). This suggests that the surface temperature response to the inclusion of surface spectral emissivity in the GCM is largely a localized thermodynamic response. Concerted large-scale dynamical responses, if any, must be small. 
(a) $\Delta T_{s}$, Mean $=0.45$, RMSE $=0.64 \mathrm{~K}$

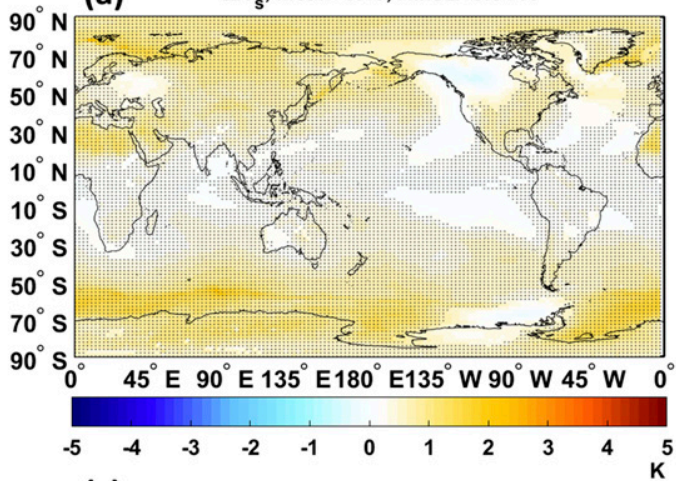

(c)

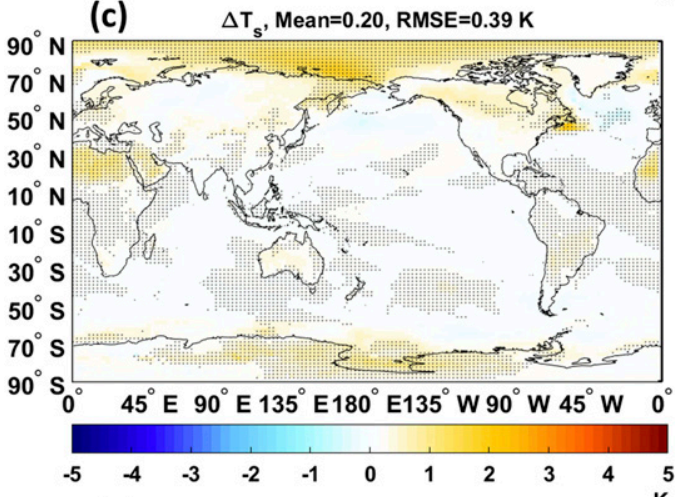

(e) $\Delta T_{s}$, Mean $=0.56$, RMSE $=2.45 \mathrm{~K}$

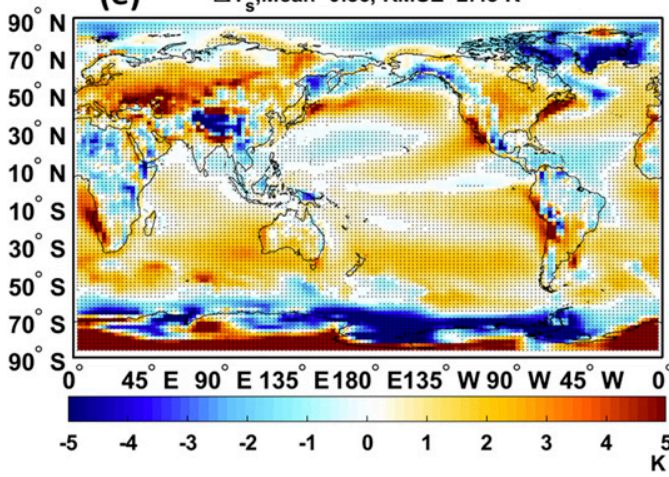

(b) $\Delta \mathrm{f}_{\text {ice }}$, Mean=-2.6e-3, RMSE $=0.015$

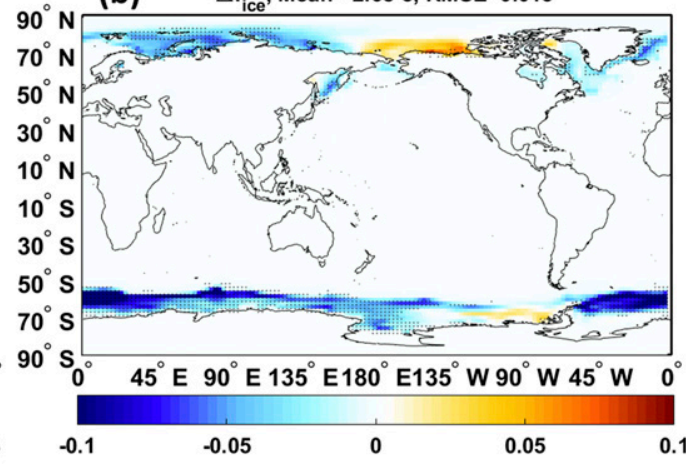

(d) $\Delta \mathrm{f}_{\text {ice }}$, Mean=-9.1e-4, RMSE $=0.009$

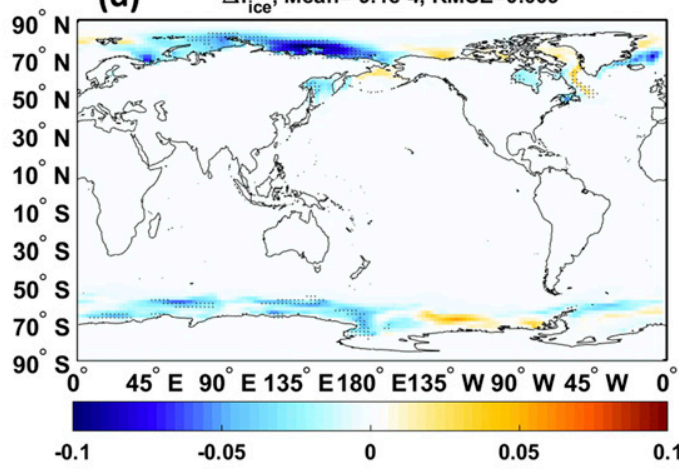

(f) $\Delta f_{\text {ice, }}$, Mean $=0.0012, R M S E=0.09$

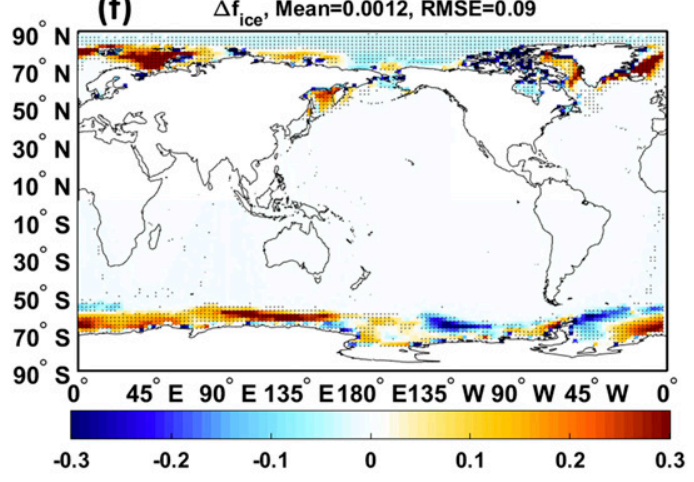

FIG. 6. (a) Difference in 30-yr mean $T_{s}$ between the modified and standard CESM simulations. Results are from the slab-ocean run and the areas with statistically significant differences are stippled (5\% significance level). (b) As in (a), but for 30-yr averages of $f_{\text {ice. }}$ (c),(d) As in (a),(b), but from the fully coupled run. (e) Difference in $T_{s}$ between the 30-yr standard CESM simulation (fully coupled run) and the observational climatology of 1961-90. The land surface temperature climatology is from Climatic Research Unit (Jones et al. 1999) and the SST climatology is from the Met Office Hadley Centre (Rayner et al. 2003). (f) Difference in $f_{\text {ice }}$ between the 30-yr standard CESM simulation (fully coupled run) and the sea ice climatology from NOAA/NSIDC (Meier et al. 2013).

Figure 6e shows the difference in surface temperature between the simulated climatology of the standard CESM (fully coupled run) and the observational climatology. The land surface air temperature climatology is from the Climatic Research Unit (Jones et al. 1999) and the SST climatology is from the Met Office Hadley Centre (Rayner et al. 2003). Comparing Figs. $6 \mathrm{c}$ and $6 \mathrm{e}$ suggests that the inclusion of surface spectral emissivity can alleviate cold biases in the standard CESM climatology for the Sahara region, the Arctic Ocean, and the Southern Ocean.

The 30-yr climatology of sea ice fraction $f_{\text {ice }}$ has noticeable differences between the standard CESM and modified CESM. As shown in Figs. 6b,d, the sea ice fraction in the modified CESM simulations tends to be less than the counterpart in the standard CESM in both hemispheres, and such differences can be as large 

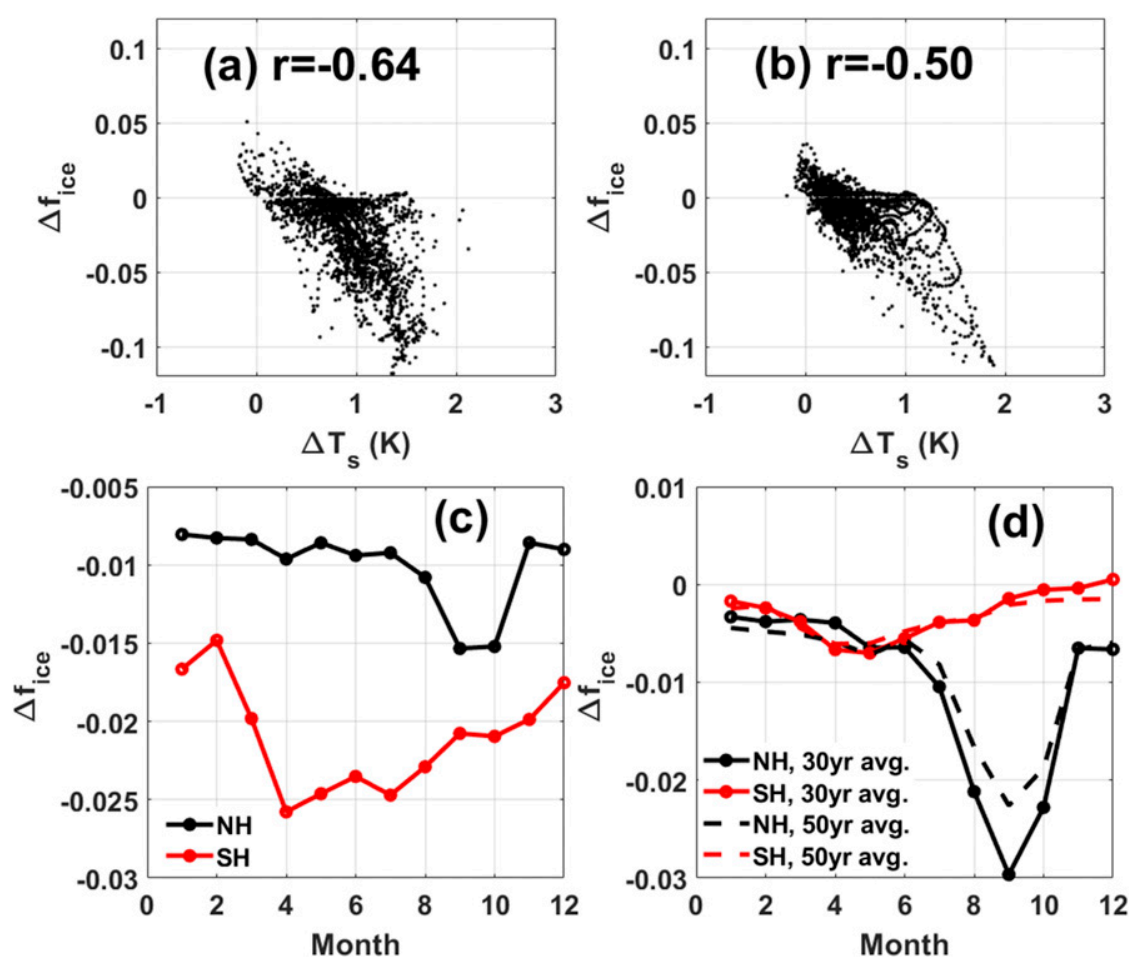

FIG. 7. (a) The scatterplot of $\Delta T_{s}$ (Fig. 6a) vs $\Delta f_{\text {ice }}$ (Fig. 6b) over sea ice regions in both hemispheres. Results are based on the slab-ocean run. (b) As in (a), but for the fully coupled run. (c) Difference in monthly mean sea ice fraction climatology between the modified and standard CESM slab-ocean runs. Black line represents the average of Northern Hemisphere high-latitude zone $\left(50^{\circ}-90^{\circ} \mathrm{N}\right)$ and red line represents the average of Southern Hemisphere high-latitude zone $\left(50^{\circ}-90^{\circ} \mathrm{S}\right)$. The average sea ice fraction is defined as sea ice fraction over the entire ocean within the high-latitude zone. (d) As in (c), but for fully coupled run. The dashed lines in (d) are results from 50 years of simulation instead of 30 years of simulation.

as -0.1 . The majority of these negative differences are also statistically significant, passing the Student's $t$ test at $5 \%$ significance level. A few patches of positive differences in sea ice fraction are seen in both hemispheres, but none of them are statistically significant. The difference in sea ice fraction is largely consistent with the difference in surface temperature change. The regions with a negative difference in sea ice fraction are also regions with a positive difference in surface temperature. The regions with a positive difference in sea ice fraction are the regions with little difference in surface temperature; moreover, neither $\Delta T_{s}$ nor $\Delta f_{\text {ice }}$ in these regions are statistically significant. In comparison to the sea ice climatology taken from NOAA/NSIDC (Meier et al. 2013), the standard CESM has positive sea ice coverage biases in both polar regions (Fig. 6f), which is consistent with the cold biases in the polar surface temperature of the standard CESM. Contrasting Figs. $6 \mathrm{~d}$ and $6 \mathrm{f}$ indicates that inclusion of surface spectral emissivity in the model can reduce the positive bias in $f_{\text {ice }}$ for both polar regions.
The difference in sea ice fraction $\Delta f_{\text {ice }}$ correlates well with $\Delta T_{s}$, as shown in Figs. 7a,b. The correlation between $\Delta T_{s}$ and $\Delta f_{\text {ice }}$ is -0.64 for the slab-ocean run and -0.50 for the fully coupled run. The value of $\Delta f_{\text {ice }}$ is not the same for each calendar month and has a noticeable seasonal dependence, as shown in Figs. 7c,d. The slab-ocean run and fully coupled run agree on the phase of $\Delta f_{\text {ice: }}$ in both hemispheres; the largest sea ice fraction difference is in fall. However, the slab-ocean and fully coupled runs disagree on the amplitude of the difference for both hemispheres. The maximum $\Delta f_{\text {ice }}$ for the slab-ocean run averaged over $50^{\circ}-90^{\circ} \mathrm{N}$ is -0.015 , whereas for the fully coupled run it is -0.03 . For $50^{\circ}-$ $90^{\circ} \mathrm{S}$, the maximum $\Delta f_{\text {ice }}$ is approximately -0.026 for the slab-ocean run and only -0.007 for the fully coupled run. Such contrast indicates the role of ocean dynamics in affecting the response of sea ice to the imposed change of surface emissivity. For the fully coupled runs, the differences in sea ice fraction and in its seasonality remain largely the same when we analyze parts of the simulation longer than 30 years. 
(a) Sahara

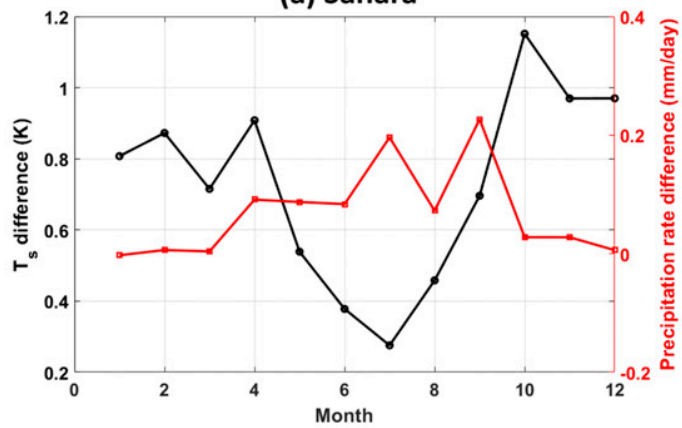

(c) Sahel

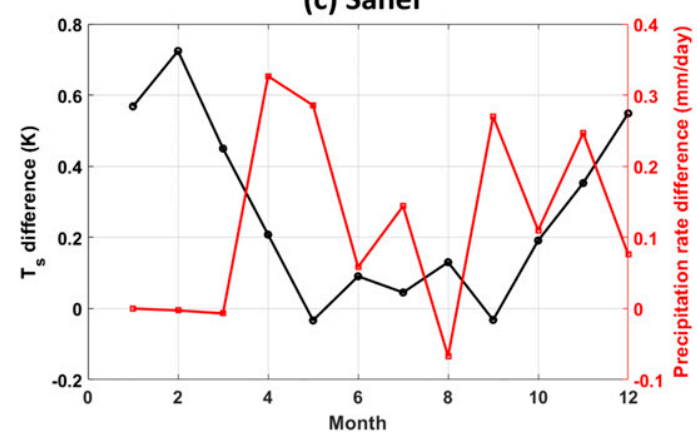

(b) Australian Deserts

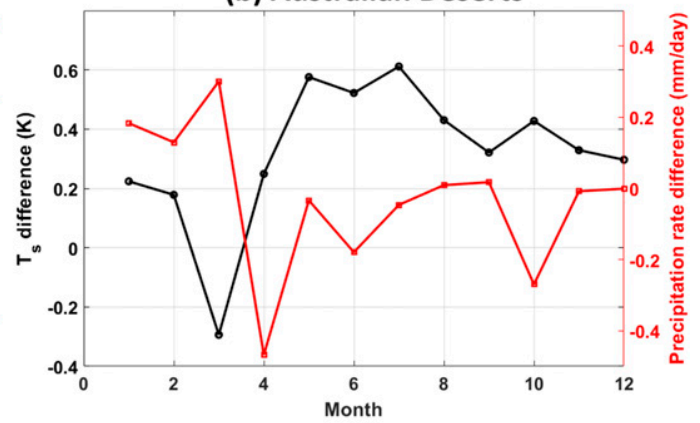

FIG. 8. (a) Difference in monthly mean $T_{s}$ (black line) and precipitation (red line) over the Sahara Desert $\left(15^{\circ}-30^{\circ} \mathrm{N}, 15^{\circ} \mathrm{W}-45^{\circ} \mathrm{E}\right)$ between the modified and standard CESM. (b) As in (a), but for the Australia desert region $\left(35^{\circ}-20^{\circ} \mathrm{S}, 122^{\circ}-142^{\circ} \mathrm{E}\right)$. (c) As in (a), but for the Sahel region $\left(5^{\circ}-15^{\circ} \mathrm{N}, 20^{\circ} \mathrm{W}-40^{\circ} \mathrm{E}\right)$.

The surface temperature and precipitation differences over the desert regions also exhibit seasonal dependence. As shown in Fig. 8, for both Sahara and Australia deserts, the surface temperature difference is generally larger in winter than in summer while the precipitation difference is opposite. Similar seasonal dependence exists in the differences for the Sahel region as well (Fig. 8c). Therefore, inclusion of more realistic surface emissivities can affect the long-term climatology as well as seasonality of the simulated surface climate, especially for the polar regions and the desert and its adjacent regions.

Our emphasis here is on the difference between the modified and standard CESM simulations. It is unlikely that surface emissivity is the largest factor affecting the discrepancies between simulated and observed climate mean states. However, as shown here, it certainly can contribute to the discrepancies between model and observation. Incorporating realistic and spectrally dependent surface emissivity into the model can improve the fidelity of the simulation and can, to some extent, alleviate biases in surface temperature and sea ice fraction in polar regions and bias in surface temperature over desert regions. Moreover, such bias reduction is expected from the physical argument described in section 2e. The largest benefit, in our opinion, is that excluding surface emissivity from the list of potential culprits for observation-model discrepancies can help expose other compensating biases hidden in the model formulations.

\section{c. Sensitivity to sea ice surface spectral emissivities}

As mentioned in section $2 \mathrm{~d}$, to understand the sensitivity of our results to different assumptions about sea ice spectral emissivities, we carry out another simulation using the modified CESM, but assuming that the spectral emissivity of sea ice is the same as that of coarse snow, instead of the emissivity of a flat ice surface. Results are shown in Fig. 9, for both the slab-ocean run and fully coupled run. Compared to the counterparts in Fig. 6, the overall patterns of $\Delta T_{s}$ and $\Delta f_{\text {ice }}$ are similar. All statistically significant changes are seen for positive difference in $\Delta T_{s}$ and negative difference in $\Delta f_{\text {ice. }}$. For certain regions in the Arctic, $\Delta T_{s}$ becomes smaller or even negative when coarse snow emissivity is used for sea ice and $\Delta f_{\text {ice }}$ changes accordingly (Fig. 9). However, $\Delta T_{s}$ and $\Delta f_{\text {ice }}$ in such regions are not statistically significant. The differences between modified and standard CESM are primarily due to the difference between spectrally dependent surface emissivities and the blackbody surface. This is why, as shown in Fig. 9, the different assumptions about the details of the spectral 

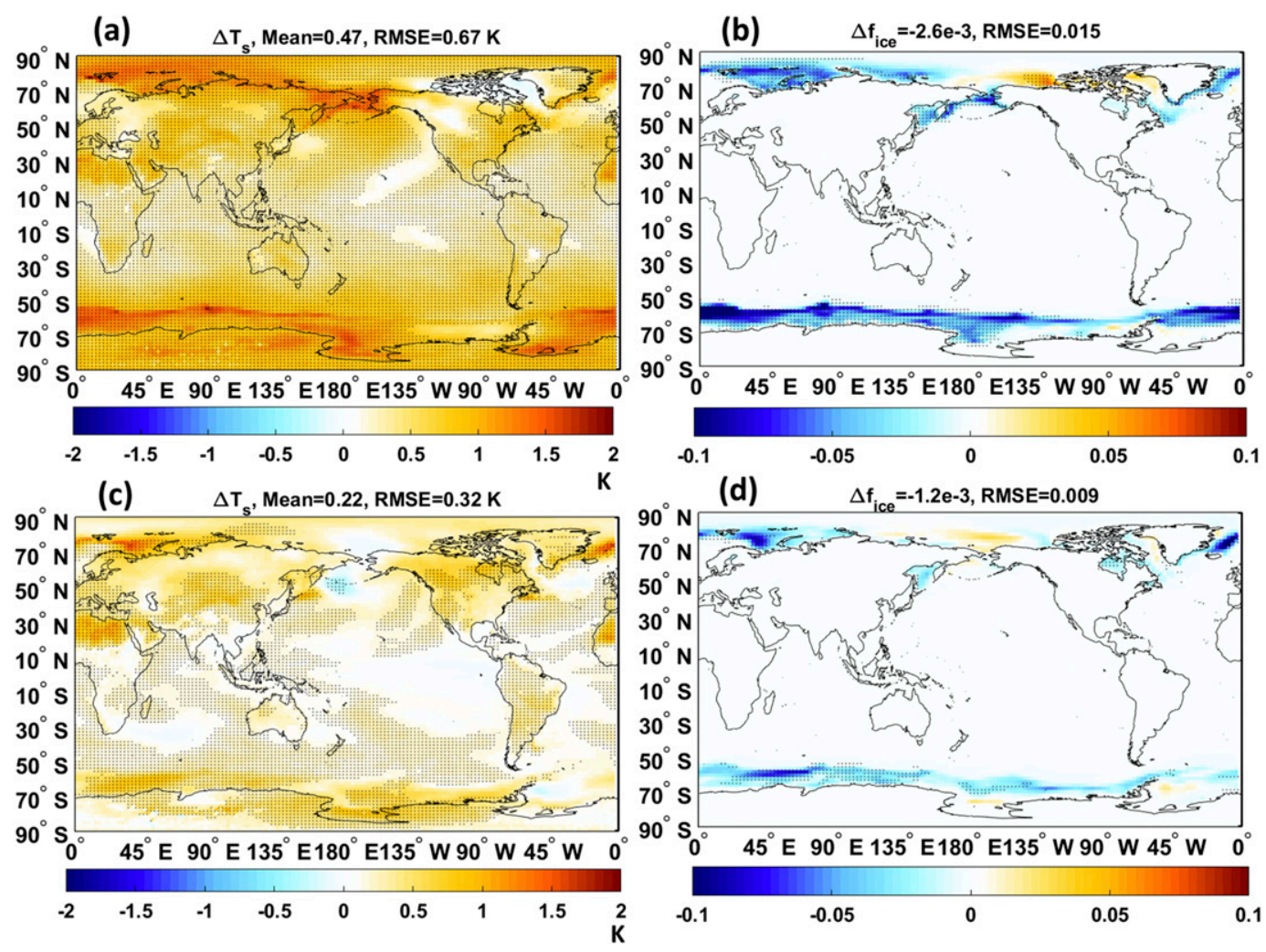

FIG. 9. (a)-(d) As in Figs. 6a-d, but the spectral emissivity of coarse snow is used instead of the emissivity of sea ice in the simulations using the modified CESM. Areas with statistically significant differences are stippled (5\% significance level).

dependence of sea ice emissivity do not fundamentally alter the main conclusions described above regarding the difference in simulated surface climatology between the modified and standard CESM models.

\section{Quantifying the impact on climate change simulation: $2 \times \mathrm{CO}_{2}$ equilibrium run}

\section{a. Motivation, experiment designs, and analysis approaches}

Differences in the shortwave reflectivity of sea ice and open water lead to well-known sea ice albedo feedback. Given the spectral emissivity difference between open ocean and ice (Fig. 1), we are naturally led to hypothesize about the existence of an ice emissivity feedback. Feldman et al. (2014) are among the first who suggested the possible existence of ice emissivity feedback using a CESM simulation with $1 \%$ annually increasing $\mathrm{CO}_{2}$ and modified surface spectral emissivities only in the far-IR.

Figures 6 and 7 indicate that an increase of surface temperatures in polar regions lead to a reduction in sea ice coverage. Then, for the portion of the spectrum where the spectral emissivity of water is larger than that of ice, the emissivity change resulting from such reduction in sea ice coverage leads to an increase of surface emission but, at the same time, a decrease of reflection of downward flux, assuming everything else is unchanged. Therefore, it is not a straightforward task to determine whether the net effect is to increase or to decrease the upward LW spectral flux. Therefore, a set of GCM experiments are needed in order to formally evaluate the sign and the strength of sea ice emissivity feedback.

Using the modified CESM, we design the following experiments to assess the sea ice emissivity feedback. To avoid any impact from the change of land surface spectral emissivity, we reset the land surface emissivity from being unity for all spectral bands in the RRTMG_LW (i.e., blackbody surface) and only allow the spectral emissivities of ocean and sea ice to change as described in section 2. Hereafter the control (CTL) run refers to the simulation with all radiative forcing agents being held constant at year 2000 levels. The $2 \times \mathrm{CO}_{2}$ run refers to the run in which the $\mathrm{CO}_{2}$ concentration is instantaneously doubled from the beginning of the simulation. Then the sea ice emissivity feedback is evaluated using the two-sided partial radiative perturbation (PRP) 

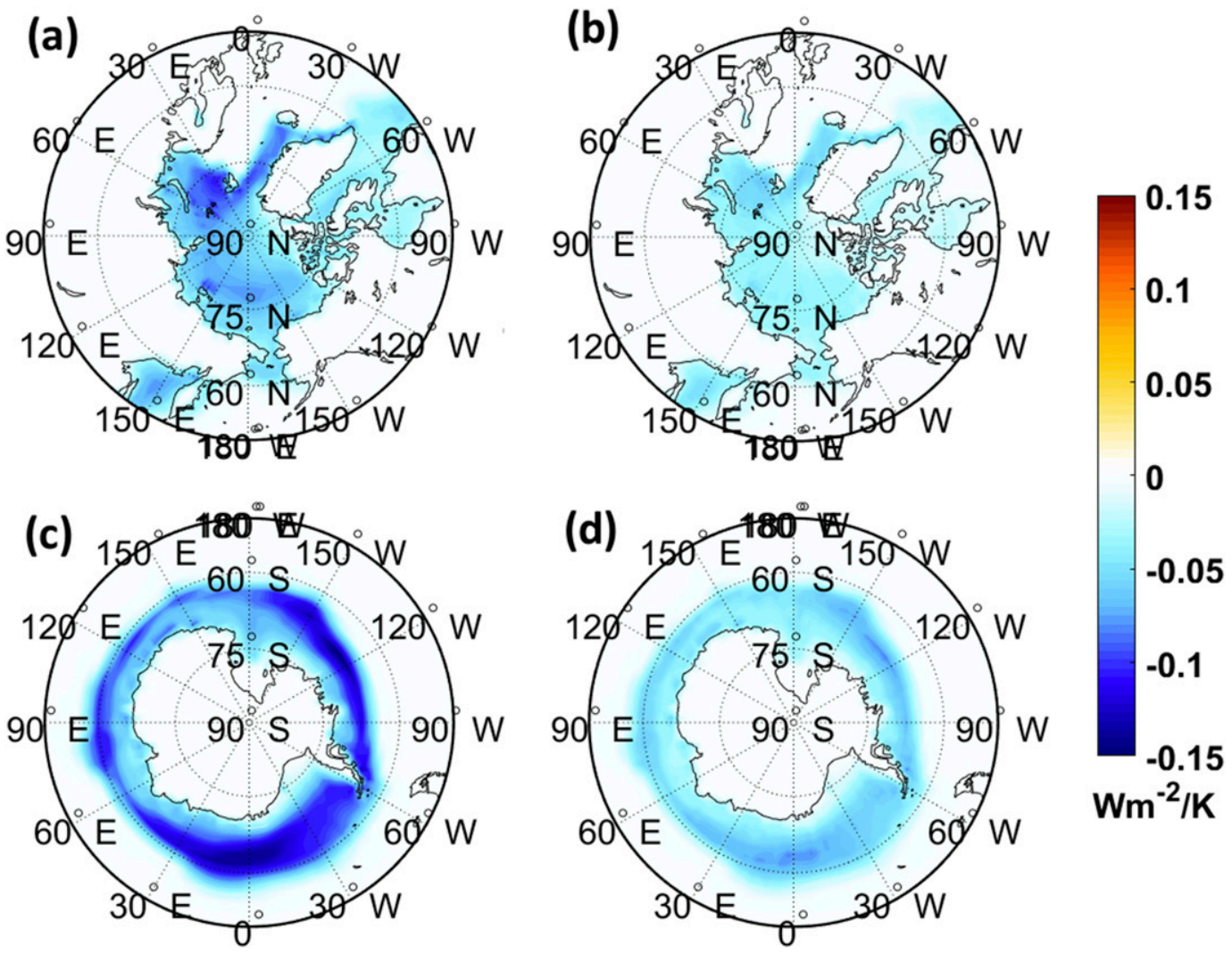

FIG. 10. The sea ice emissivity feedback as derived from CTL and $2 \times \mathrm{CO}_{2}$ run using the two-sided PRP method. The results are based on the slab-ocean runs as described in section 4. The spectral emissivity of flat ice surface is used here as sea ice emissivity. Both clear-sky and all-sky feedbacks are shown: (a) Arctic clear sky, (b) Arctic all sky, (c) Antarctic clear sky, and (d) Antarctic all sky.

method (Wetherald and Manabe 1988). Specifically, the monthly mean sea ice coverage from 30 years of $2 \times \mathrm{CO}_{2}$ run and the monthly mean atmospheric states from $30-\mathrm{yr}$ output of the control run are fed into an offline radiative transfer model to compute the OLR. The difference of the OLR computed in this manner and the OLR from the control run is deemed as the perturbation to OLR resulting from sea ice emissivity feedback in response of the doubling of $\mathrm{CO}_{2}$. This is normally referred as a forward PRP calculation. Then, a backward PRP calculation is performed by replacing the monthly mean sea ice coverage in the $2 \times \mathrm{CO}_{2}$ run with the sea ice coverage in the control run and the perturbation to the OLR from the $2 \times \mathrm{CO}_{2}$ run is computed accordingly. The sea ice emissivity feedback is then estimated as the average of results from both forward and backward PRP calculations divided by the change of global-mean surface temperature between the $2 \times \mathrm{CO}_{2}$ run and the control run, often referred as a two-sided PRP calculation. The offline radiative transfer model used in the evaluation is described in detail by Chen et al. (2013) and Liu et al. (2006). It is based on the Principal Component-Based
Radiative Transfer Model (PCRTM) developed by Liu et al. (2006). The subgrid variability of cloud is then taken into account to ensure maximum consistency with the cloud-radiation scheme used in the GCM. The model has been benchmarked with a line-by-line radiative transfer model to show its accuracy and computational speed. It has been checked against the GCM-simulated OLR to validate that the simulation of cloud subgrid variability is done in a way consistent with the GCM radiation scheme (Chen et al. 2013). The PCRTM model has been used in other published studies such as Huang et al. (2014) and Bantges et al. (2016). The model is used here to compute OLR as well as spectrally resolved flux.

\section{b. Estimation of sea ice emissivity feedback}

As mentioned above, the simulations and feedback calculations are done for two separate sets: one set assumes the spectral emissivity of sea ice to be that of flat ice, and the other set assumes it to be the same as coarse snow. Figure 10 shows the broadband sea ice emissivity feedbacks derived from the slab-ocean runs when the spectral emissivity of flat ice is used. The sea ice 

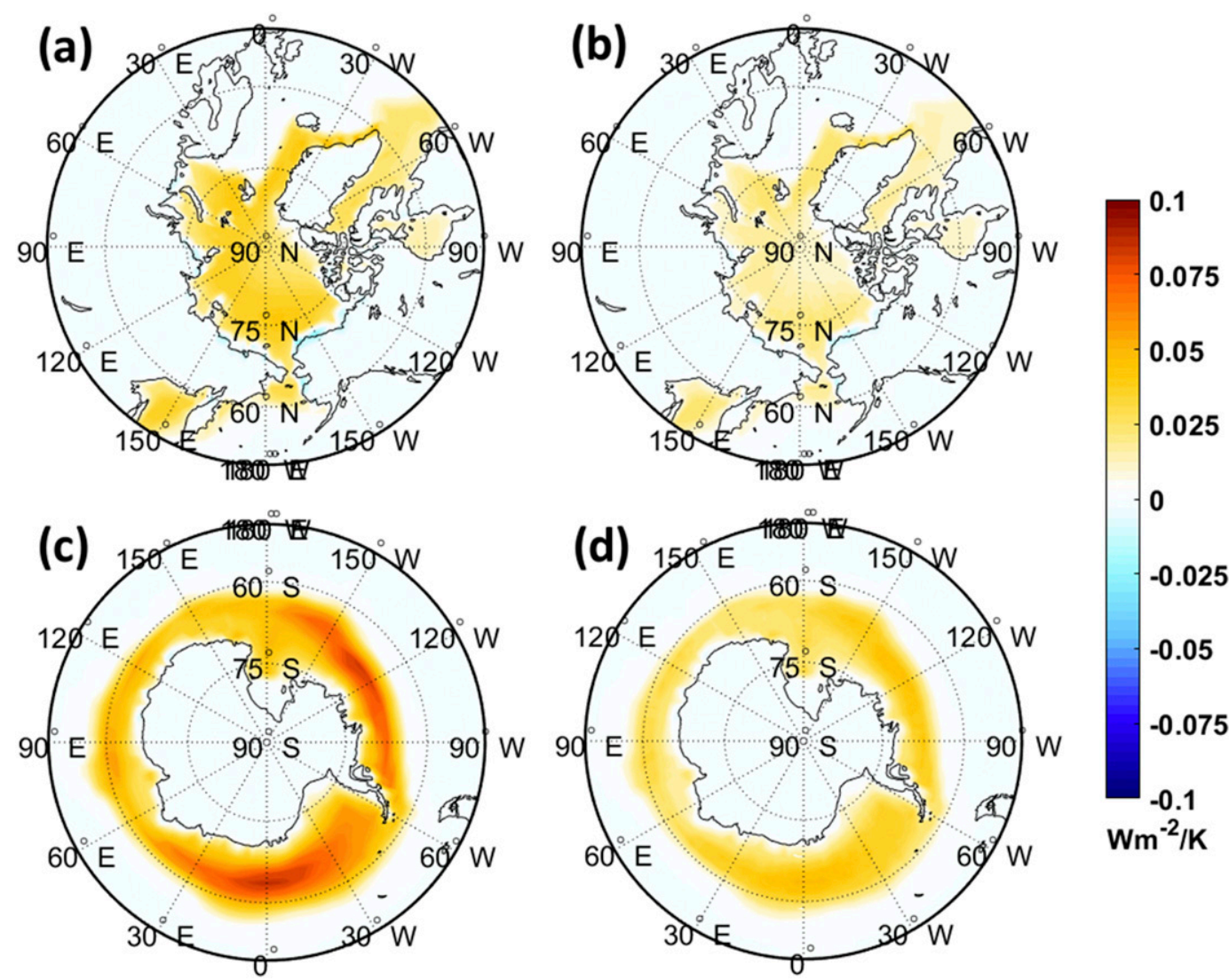

FIG. 11. As in Fig. 10, but the spectral emissivity of coarse snow is used instead of the emissivity of sea ice.

emissivity feedback is slightly negative for both the Arctic and Antarctic. Globally averaged all-sky sea ice emissivity feedback is only $-0.003 \mathrm{~W} \mathrm{~m}^{-2} \mathrm{~K}^{-1}$. The clear-sky sea ice emissivity feedback is $-0.007 \mathrm{~W} \mathrm{~m}^{-2} \mathrm{~K}^{-1}$. Results from the fully coupled runs are similar, with the all-sky and clear-sky sea ice emissivity feedback being -0.003 and $-0.006 \mathrm{~W} \mathrm{~m}^{-2} \mathrm{~K}^{-1}$, respectively (figures not shown here). The sea ice emissivity feedback derived from the slab-ocean simulations that assume sea ice emissivity as coarse snow emissivity is shown in Fig. 11. The feedback is slightly positive for both polar regions. Globally averaged all-sky sea ice emissivity feedback is $0.002 \mathrm{~W} \mathrm{~m}^{-2} \mathrm{~K}^{-1}$ and clear-sky sea ice emissivity feedback is $0.003 \mathrm{~W} \mathrm{~m}^{-2} \mathrm{~K}^{-1}$.

In contrast, estimated surface shortwave albedo feedback using the broadband radiative kernel technique (Soden et al. 2008) is $0.30 \mathrm{~W} \mathrm{~m}^{-2} \mathrm{~K}^{-1}$ for the fully coupled simulations. This estimate is consistent with the range of surface albedo feedbacks by the CMIP3 and CMIP5 GCMs (Soden et al. 2008; Sanderson et al. 2010; Vial et al. 2013). Therefore, the sea ice emissivity feedback strength examined here is two orders of magnitude smaller than that of surface albedo feedback, for both sets of sea ice emissivities used in the simulations.

The spectrally resolved sea ice emissivity feedbacks are shown in Fig. 12. When the emissivity of flat ice surface is assumed for sea ice emissivity, the broadband sea ice emissivity feedback is negative and, spectrally, the largest contribution comes from the mid-IR spectral region around $740-940 \mathrm{~cm}^{-1}$ (Fig. 12). This spectral region is where the spectral emissivity of open water is larger than that of flat ice surface as shown in Fig. 1. The spectral region intersects with the mid-IR window region. As explained in section $2 \mathrm{e}$, the atmospheric opacity is small over the mid-IR window region and thus the downward flux at surface is small. As a result, the change of surface emission significantly dominates over the change of reflection of downward flux and the feedback is negative over the spectral region. The feedback is only slightly positive in the dirty window of the far-IR (i.e., $450-600 \mathrm{~cm}^{-1}$ ). The spectral emissivity of flat ice surface is larger than that of open water (Fig. 1) in this portion of the spectrum but the optical depth of atmosphere is larger in this region than in the mid-IR window region. As a result, the compensation 


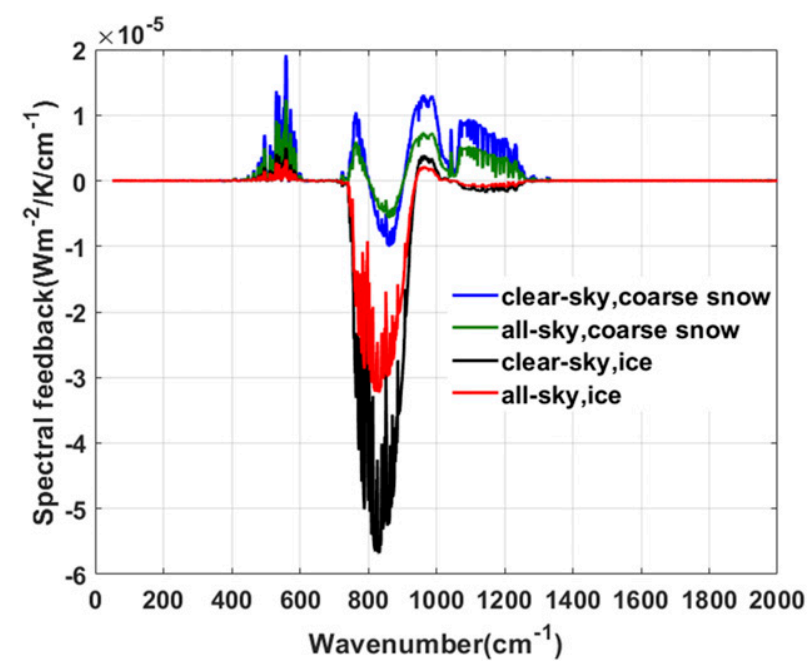

FIG. 12. The spectrally resolved all-sky and clear-sky sea ice emissivity feedback based on slab-ocean runs. In the figure legend "ice" refers to the runs that assume the flat ice emissivity as the sea ice.

between the surface emission and reflection of downward spectral flux only leads to a slightly positive feedback over this spectral region.

When coarse snow emissivity is as assumed for sea ice emissivity, it is larger than the water emissivity and the deviation can be twice as great as that between water and flat ice emissivity (Fig. 1). As a result, a larger positive feedback can be seen over the far-IR dirty window. Over the mid-IR spectral region, the coarse snow emissivity is larger than the water emissivity, except over 800$900 \mathrm{~cm}^{-1}$, where the former is only slightly smaller than the latter (Fig. 1). Thus, small negative feedbacks are expected between 800 and $900 \mathrm{~cm}^{-1}$ and positive feedbacks are seen for the rest of the mid-IR spectral region (Fig. 12). Integrating over the entire LW spectrum then leads to a positive feedback as shown in Fig. 11.

The analysis here shows that the LW sea ice emissivity feedback can be negative or positive, depending on the assumption about the spectral emissivity of sea ice. However, its strength is two orders of magnitude smaller than that of surface albedo feedback for either case. Moreover, we use a broadband radiative kernel (Soden et al. 2008) to compute other radiative feedback strengths (i.e., water vapor feedbacks, lapse rate feedback, and sea ice albedo feedback) and use the adjustment method described by Soden et al. (2008) to compute cloud feedbacks. We verify that these radiative feedback strengths change little from results derived from the standard CESM. Therefore, including the sea ice emissivity feedback will have little impact on the simulated climate change and the estimated climate sensitivity. In fact, if we define $\Delta T_{s}$ as the $20-\mathrm{yr}$ global- mean temperature difference between the $2 \times \mathrm{CO}_{2}$ equilibrium run and the control run, $\Delta T_{s}$ from the fully coupled runs using the modified CESM is only $0.06 \mathrm{~K}$ lower than that derived using the standard CESM.

\section{Conclusions and discussion}

Incorporating the surface spectral emissivity into the atmosphere model of the CESM substantially improves the fidelity of the radiative representation of the atmospheric lower boundary. By carefully ensuring consistency of the broadband longwave flux across different models in the CESM, TOA energy balance in the modified CESM can be reached without retuning the model. The impacts on the simulated global-mean surface energy budget are mainly manifested as a decrease of net upward longwave flux at the surface and a comparable increase of latent heat flux. The global-mean surface temperature difference between the modified and standard CESM simulations is $0.20 \mathrm{~K}$ for the fully coupled run and $0.54 \mathrm{~K}$ for the slab-ocean run. The modified CESM simulation exhibits higher surface temperature than the standard CESM in the polar regions of both hemispheres, as well as in desert regions, especially the Sahara Desert. Accordingly, the climatological mean sea ice fraction in the modified CESM simulation is less than the counterpart in the standard CESM by as much as 0.1. Given the discrepancies between the standard CESM simulations and observational climatologies, such differences between the modified and standard CESM suggest that incorporating surface spectral emissivity into the model can help reduce biases in the simulated surface temperatures in high-latitude regions and in the Sahara Desert, as well as biases in the simulated sea ice fractions.

By allowing the surface spectral emissivity to be a prognostic variable over the sea ice regions and fixing it over lands, the strength of ice emissivity feedback is evaluated using the two-sided PRP method applied to the $2 \times \mathrm{CO}_{2}$ run and the control run of present climate. The sign of feedback depends on the spectral details of assumed sea ice emissivity. But in either case the strength of ice emissivity feedback is two orders of magnitude smaller than that of surface albedo feedback. Inclusion of surface emissivity also has little impact on the strength of other radiative feedbacks in the CESM and thus leads to little change in the simulated global surface temperature in response to the increase of $\mathrm{CO}_{2}$.

The analysis in this study mainly focuses on the global scale and on surface climate variables. The impact of inclusion of surface spectral emissivity on simulated regional climate (e.g., over the Sahara Desert region) could be significant and not only limited to surface variables (Chen et al. 2016). Moreover, the land model in the CESM employs different assumptions about the surface emissivities from 


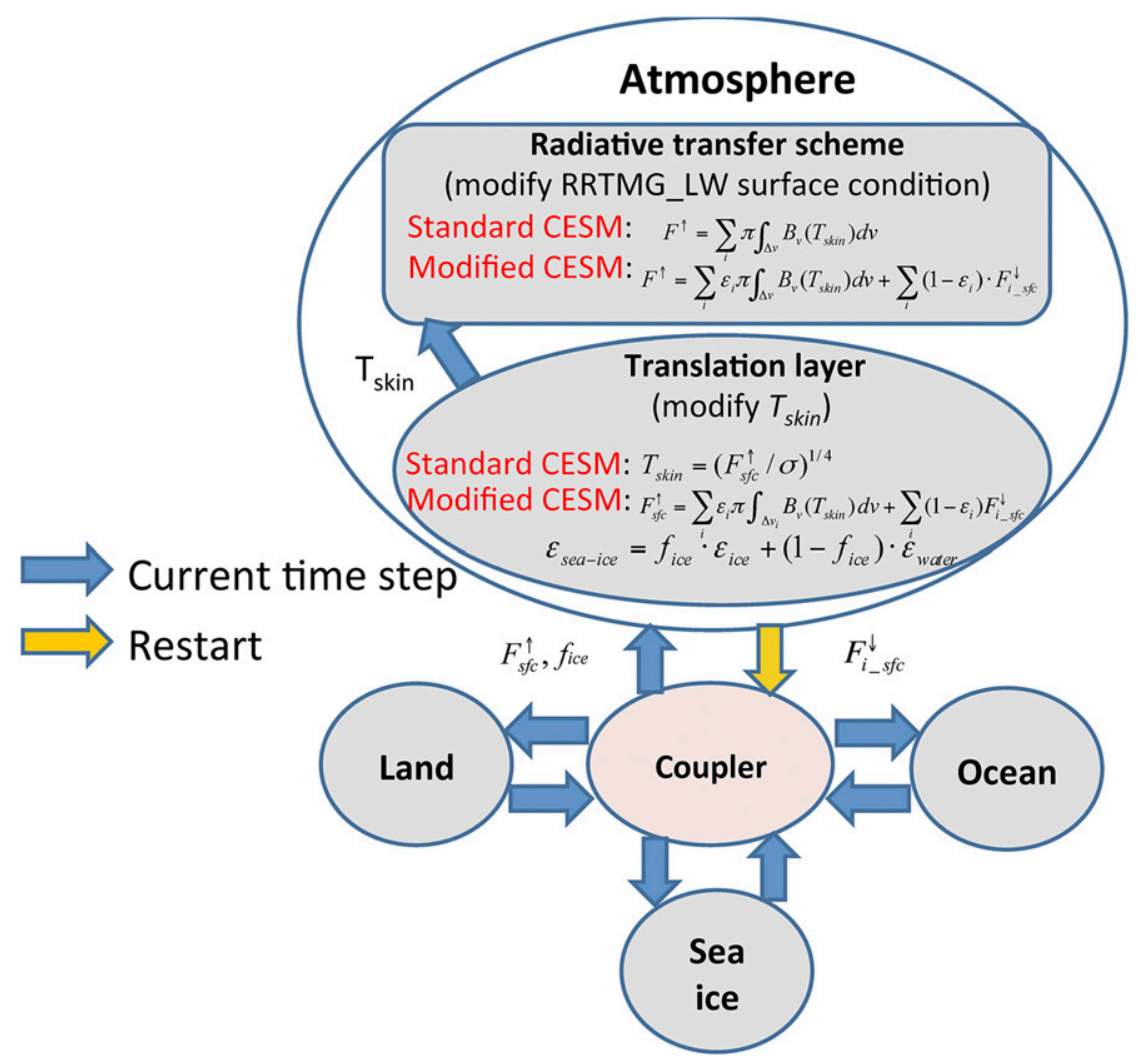

FIG. A1. A schematic diagram to describe our modification to incorporate the surface spectral emissivity into the CESM atmosphere model. The definitions of symbols are as in the text.

the atmosphere model. Future work would be needed to update such treatment in the land, ice, and ocean models by incorporating more realistic and spectrally dependent surface emissivities. Only by doing so, can the ultimate consistency across atmosphere and surface models within a GCM be assured and the radiative coupling between the atmosphere and surface be modeled with more fidelity.

Acknowledgments. We are grateful to three reviewers and the editor, Dr. Shell, for their insightful comments, which greatly improves the quality and clarity of the article. The CESM code is obtained from https://svn-ccsm-release.cgd. ucar.edu/model_versions/cesm1_1_1. This material is based upon work supported by the U.S. Department of Energy, Office of Science, Office of Biological and Environmental Research, Climate and Environmental Science Division under Award DE-SC0012969 to the University of Michigan and Award DE-SC0013080 to Texas A\&M University.

\section{APPENDIX}

\section{Modification to the Standard CESM}

Using a schematic diagram, we summarize how we include the surface spectral emissivity into the modified
CESM in Fig. A1. The LW radiation scheme in the atmosphere model of the CESM, RRTMG_LW, takes surface radiative skin temperature $T_{\text {skin }}$ as an input variable. That is why in the translation layer we compute $T_{\text {skin }}$ using the broadband LW flux passed from the coupler and the surface spectral emissivity as used in the RRTMG_LW. Then $T_{\text {skin }}$ is fed into the RRTMG_LW that incorporated same surface spectral emissivity in the radiative transfer calculation. (Further details of the coupler for the standard default configuration in the CESM1.1 can be found in http:// www.cesm.ucar.edu/models/cesm1.2/cpl7/coupler_flow. pdf. In particular, the sequence of events implemented within each driver time loop of the coupler processors can be found in chapter 3 of CPL7 User's Guide (Craig 2011).

\section{REFERENCES}

Baldridge, A. M., S. J. Hook, C. I. Grove, and G. Rivera, 2009: The ASTER spectral library version 2.0. Remote Sens. Environ., 113, 711-715, https://doi.org/10.1016/j.rse.2008.11.007.

Bantges, R., H. Brindley, X. H. Chen, X. L. Huang, J. Harries, and J. Murray, 2016: On the detection of robust multidecadal changes in the Earth's outgoing longwave radiation spectrum. J. Climate, 29, 4939-4947, https://doi.org/ 10.1175/JCLI-D-15-0713.1. 
Chen, X. H., X. L. Huang, and X. Liu, 2013: Non-negligible effects of cloud vertical overlapping assumptions on longwave spectral fingerprinting studies. J. Geophys. Res. Atmos., 118, 73097320, https://doi.org/10.1002/jgrd.50562.

,-- , and M. G. Flanner, 2014: Sensitivity of modeled far-IR radiation budgets in polar continents to treatments of snow surface and ice cloud radiative properties. Geophys. Res. Lett., 41, 6530-6537, https://doi.org/10.1002/2014GL061216.

Chen, Y.-H., X. H. Chen, X. L. Huang, and M. G. Flanner, 2016: The effects of surface longwave emissivity on atmospheric circulation and convection at Sahara and Sahel regions. 2016 Fall Meeting, San Francisco, CA, Amer. Geophys. Union, Abstract A31F-0101.

Cheng, H. Z., X. H. Chen, and X. L. Huang, 2016: Quantification of the errors associated with the representation of surface emissivity in the RRTMG_LW. J. Quant. Spectrosc. Radiat. Transfer, 180, 167-176, https://doi.org/10.1016/j.jqsrt.2016.05.004.

Clough, S. A., M. W. Shephard, E. J. Mlawer, J. S. Delamere, M. Iacono, K. Cady-Pereira, S. Boukabara, and P. D. Brown, 2005: Atmospheric radiative transfer modeling: A summary of the AER codes. J. Quant. Spectrosc. Radiat. Transfer, 91, 233244, https://doi.org/10.1016/j.jqsrt.2004.05.058.

Craig, A. P., 2011: CPL7 user's guide. Accessed 30 March 2018, http:// www.cesm.ucar.edu/models/cesm1.2/cpl7/doc/book1.html.

Feldman, D. R., W. D. Collins, R. Pincus, X. L. Huang, and X. H. Chen, 2014: Far-infrared surface emissivity and climate. Proc. Natl. Acad. Sci. USA, 111, 16297-16302, https://doi.org/ 10.1073/pnas.1413640111.

Gent, P. R., and Coauthors, 2011: The Community Climate System Model version 4. J. Climate, 24, 4973-4991, https://doi.org/ 10.1175/2011JCLI4083.1.

Huang, X. L., X. H. Chen, B. J. Soden, and X. Liu, 2014: The spectral dimension of longwave feedback in the CMIP3 and CMIP5 experiments. Geophys. Res. Lett., 41, 7830-7837, https://doi.org/10.1002/2014GL061938.

,$- \ldots$, D. Zhou, and X. Liu, 2016: An observationally based global band-by-band surface emissivity dataset for climate and weather simulations. J. Atmos. Sci., 73, 3541-3555, https://doi.org/ 10.1175/JAS-D-15-0355.1.

Jones, P. D., M. New, D. E. Parker, S. Martin, and I. G. Rigor, 1999: Surface air temperature and its variations over the last 150 years. Rev. Geophys., 37, 173-199, https://doi.org/10.1029/ 1999RG900002.

Liu, X., W. L. Smith, D. K. Zhou, and A. Larar, 2006: Principal component-based radiative transfer model for hyperspectral sensors: Theoretical concept. Appl. Opt., 45, 201-209, https:// doi.org/10.1364/AO.45.000201.

Meier, W., F. Fetterer, M. Savoie, S. Mallory, R. Duerr, and J. Stroeve, 2013: NOAA/NSIDC climate data record of passive microwave sea ice concentration, version 2. National
Snow and Ice Data Center, NOAA/National Climatic Data Center, NCDC Satellite Climate Data Record Program, Boulder, CO, accessed 23 January 2017, https://doi.org/ 10.7265/N55M63M1.

Mlawer, E. J., S. J. Taubman, P. D. Brown, M. J. Iacono, and S. A. Clough, 1997: Radiative transfer for inhomogeneous atmospheres: RRTM, a validated correlated-k model for the longwave. J. Geophys. Res., 102, 16663-16 682, https://doi.org/ 10.1029/97JD00237.

Neale, R. B., and Coauthors, 2012: Description of the NCAR Community Atmosphere Model (CAM 5.0). NCAR Tech. Note NCAR/TN-486+ STR, 274 pp., http://www.cesm.ucar. edu/models/cesm1.0/cam/docs/description/cam5_desc.pdf.

Petrich, C., and H. Eicken, 2009: Growth, structure and properties of sea ice. Sea Ice, 2nd ed. D. N. Thomas and G. S. Dieckmann, Eds., Wiley-Blackwell, 23-77, https://doi.org/10.1002/ 9781444317145.ch2

Rayner, N. A., D. E. Parker, E. B. Horton, C. K. Folland, L. V. Alexander, D. P. Rowell, E. C. Kent, and A. Kaplan, 2003: Global analyses of sea surface temperature, sea ice, and night marine air temperature since the late nineteenth century. J. Geophys. Res., 108, 4407, https://doi.org/10.1029/ 2002JD002670.

Sanderson, B. M., K. M. Shell, and W. Ingram, 2010: Climate feedbacks determined using radiative kernels in a multithousand member ensemble of AOGCMs. Climate Dyn., 35, 1219-1236, https://doi.org/10.1007/s00382-009-0661-1.

Seemann, S. W., E. E. Borbas, R. O. Knuteson, G. R. Stephenson, and H.-L. Huang, 2008: Development of a global infrared land surface emissivity database for application to clear sky sounding retrievals from multispectral satellite radiance measurements. J. Appl. Meteor. Climatol., 47, 108-123, https:// doi.org/10.1175/2007JAMC1590.1.

Soden, B. J., I. M. Held, R. Colman, K. M. Shell, J. T. Kiehl, and C. A. Shields, 2008: Quantifying climate feedbacks using radiative kernels. J. Climate, 21, 3504-3520, https://doi.org/ 10.1175/2007JCLI2110.1.

Vial, J., J. L. Dufresne, and S. Bony, 2013: On the interpretation of inter-model spread in CMIP5 climate sensitivity estimates. Climate Dyn., 41, 3339-3362, https://doi.org/10.1007/s00382013-1725-9.

Wetherald, R., and S. Manabe, 1988: Cloud feedback processes in a general circulation model. J. Atmos. Sci., 45, 13971416, https://doi.org/10.1175/1520-0469(1988)045<1397: CFPIAG $>2.0 . \mathrm{CO} ; 2$.

Zhou, D. K., A. M. Larar, X. Liu, W. L. Smith, L. L. Strow, P. Yang, P. Schlüssel, and X. Calbet, 2011: Global land surface emissivity retrieved from satellite ultraspectral IR measurements. IEEE Trans. Geosci. Remote Sens., 49, 1277-1290, https://doi.org/ 10.1109/TGRS.2010.2051036. 